\title{
Ultrafast imaging Raman spectroscopy of large-area samples without stepwise scanning
}

\author{
Elmar Schmälzlin $^{1}$, Benito Moralejo ${ }^{1}$, Daniel Bodenmüller ${ }^{1}$, Maxim E. Darvin ${ }^{2}$, Gisela Thiede ${ }^{2}$, and \\ Martin M. Roth ${ }^{1}$ \\ ${ }^{1}$ Leibniz-Institut für Astrophysik Potsdam (AIP), Potsdam, 14482, Germany \\ ${ }^{2}$ Center of Experimental and Applied Cutaneous Physiology, Department of Dermatology, Venerology and \\ Allergology, Charité - Universitätsmedizin Berlin, Berlin, 10117, Germany \\ Correspondence to: Elmar Schmälzlin (eschmaelzlin@aip.de)
}

Received: 9 February 2016 - Revised: 20 May 2016 - Accepted: 23 June 2016 - Published: 13 July 2016

\begin{abstract}
Step-by-step, time-consuming scanning of the sample is still the state-of-the-art in imaging Raman spectroscopy. Even for a few 100 image points the measurement time may add up to minutes or hours. A radical decrease in measurement time can be achieved by applying multiplex spectrographs coupled to imaging fiber bundles that are successfully used in astronomy. For optimal use of the scarce and expensive observation time at astronomical observatories, special high-performance spectrograph systems were developed. They are designed for recording thousands of spatially resolved spectra of a two-dimensional image field within one single exposure. Transferring this technology to imaging Raman spectroscopy allows a considerably faster acquisition of chemical maps. Currently, an imaging field of up to $1 \mathrm{~cm}^{2}$ can be investigated. For porcine skin the required measurement time is less than $1 \mathrm{~min}$. For this reason, this technique is of particular interest for medical diagnostics, e.g., the identification of potentially cancerous abnormalities of skin tissue.
\end{abstract}

1

\section{Introduction}

When monochromatic light impinges on a sample, the inelastically scattered light is wavelength shifted (Raman effect). The shift contains information about the vibration states of the impinged molecules and any crystal structures. Raman spectra are fingerprints, which allow a contact- and label-free identification of chemical structures. The underlying physical effect was already discovered in 1928 by Chandrasekhara Venkata Raman. Unfortunately, Raman lines are extremely faint (only a fraction of $1 \times 10^{-7}$ of the scattered light is Raman scattering). For this reason, the number of practical applications of Raman spectroscopy was for a long time rather limited. However, based on considerable progress in laser and detector technologies, the situation has changed completely. In particular, in the fields of medical diagnostics and life sciences, Raman microscopes have become more and more common. In contrast to the traditional infrared absorption spectroscopy, Raman spectroscopy is not impeded by the infrared light absorption of water. Raman lines ap- pear relative to the laser excitation wavelength. When using excitation light at visible wavelengths, the Raman lines always appear in a range where the absorption of water is negligibly small. The Raman activity of water is also small. Moreover, in the majority of cases the spectral positions of water Raman signals are outside of the region of interest. Thus, Raman spectroscopy works well in aqueous environments. This turns out to be a key advantage with regard to biological samples. Reviews of methods and applications of Raman imaging are given in Opilik et al. (2013) and Stewart et al. (2012). In the field of medical diagnostics, imaging Raman spectroscopy is a promising technique for identifying cancerous parts of tissue (Reble et al., 2014; Zhao et al., 2015). A Raman microscope with a special design for the examination of skin in vivo on the patient is already commercially available (gen2-SCA Raman system for in vivo skin analysis. RiverD International B.V., Rotterdam, the Netherlands, http://www.riverd.com, last access 18 May 2016).

However, like common Raman microscopes, this instrument can only detect Raman scattering arising from a single 
spot with a diameter in the range of micrometers. By contrast, the diagnostics of potentially cancerous skin areas must be performed in the range of at least some square millimeters. Standard point-by-point scanning is time-consuming. Even for an image being composed of some 100 pixels, the measurement time can exceed $1 \mathrm{~h}$. For examination at a patient or for fast-changing samples, this time span is far too long to be practical. To accelerate the measuring procedure, various techniques for a parallel data collection are described (Li et al., 2013; Hagen et al., 2012). Of particular interest are full-throughput snapshot techniques, also called multichannel or integral field spectroscopy (IFS). They work without any tuning procedures and fundamentally sacrifice no light during the recording procedure. In the field of astronomy, multi-channel spectroscopy techniques have been developed since more than 3 decades ago (Vanderriest, 1980) to record images of faint galaxies that are both spatially and spectrally resolved, and that are obtained with one single exposure. By avoiding any scanning procedure, the expensive observation time at large telescopes is thus utilized more efficiently. A review of the breakthrough obtained with this technique can be found in Roth (2010). The currently most powerful spectrograph, MUSE (Multi-Unit Spectroscopic Explorer, Bacon et al., 2004, http://www.eso.org/sci/facilities/ paranal/instruments/muse.html, last access on 18 May 2016), was commissioned at the Very Large Telescope observatory in Paranal, Chile, in spring 2014.

MUSE consists of 24 connected spectrograph modules and is capable of acquiring an image field of the sky with a size of $1 \mathrm{arcmin}^{2}$, a sampling of $0.2 \times 0.2 \mathrm{arsec}^{2}$, i.e., a total number of 90000 spatial elements ("spaxels"), and a depth of 4000 spectral bins within one single exposure. From every single spaxel the entire spectrum from 465 to $930 \mathrm{~nm}$ can be recorded at a spectral resolution of $0.25 \mathrm{~nm}$. As part of a technology transfer project, a 25th MUSE spectrograph module was procured and installed in an AIP laboratory (Roth et al., 2012). The aim of the project is to investigate the suitability of fiber-coupled IFS for the identification of skin abnormalities including cancerous areas by means of imaging Raman spectroscopy. With regard to patient friendliness, the measurement times should be kept as short as possible.

\section{Materials and methods}

\subsection{Fiber bundle-coupled astronomy spectrograph}

The basic idea of IFS is slicing a two-dimensional image into single stripes and assembling them longitudinally in front of the entrance slit of a spectrograph (Allington-Smith, 2006). Amongst other techniques, this can be performed by an image slicer, i.e., a mirror stack followed by a recombining optic, or by use of a fiber bundle. Fiber bundle-coupled IFS is also called FAST (fiber array spectral translation, Stewart et al., 2012) or FRIS (fiber-reformatting imaging spectroscopy, Hagen et al., 2012). Figure 1 describes the concept. At the

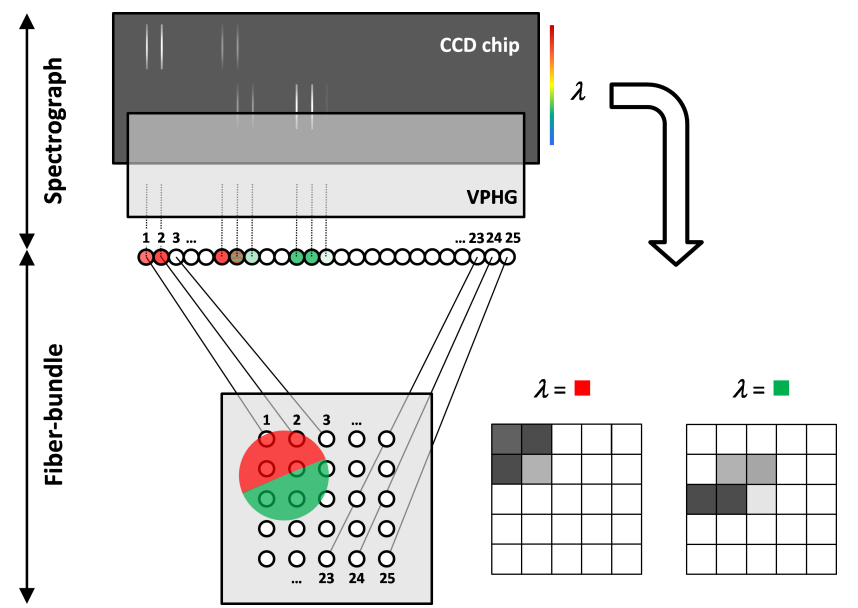

Figure 1. Function principle of fiber bundle-coupled IFS. The image of the sample (red and green semi-circles) is focused on the fiber matrix of the image acquisition head. At the spectrograph side, the fibers are arranged in a one-dimensional row. After passing a spectral disperser (e.g., a volume phase holographic grating, VPHG), every fiber produces an individual intensity- and spectrum-dependent light trace at the CCD chip. The raw signal of the CCD is evaluated by software and converted into a data cube that contains the entire spectral and spatial information.

sample side, the image of the investigated object, which may also be a Raman or fluorescence image, is focused at the surface of a two-dimensional fiber array. The fibers collect the signal of the image. At the spectrograph side, the fibers of the bundle are arranged in one row forming a pseudo slit for the spectrograph. The signals emerging from each fiber are collimated, pass through a dispersive element, and finally form spectrally dispersed light traces on a charge-coupled device (CCD) chip. Data reduction software extracts and calibrates the CCD raw signal and finally produces a data cube that contains the entire spectral and spatial information. The setup works in essence as if every single fiber were connected to an individual single-channel spectrograph.

The employed astronomy spectrograph and the fiber bundle were presented previously (Schmälzlin et al., 2014). In brief: the MUSE spectrograph unit is a high-performance fully refractive optical system that covers a spectral range from 465 to $930 \mathrm{~nm}$ with about $0.25 \mathrm{~nm}$ resolution. The size of one unit including the CCD camera housing is approximately $0.6 \mathrm{~m} \times 1.9 \mathrm{~m} \times 0.6 \mathrm{~m}$. It comprises a volume phase holographic grating (VPHG) and a liquid-nitrogen cooled $4 \mathrm{~K} \times 4 \mathrm{~K}$ pixel $\mathrm{CCD}$ detector system. The VPGH, consisting of a phase-modulating dichromatic gelatin layer that is sealed between two glass plates, shows up to $80 \%$ diffraction efficiency and minimally scattered light over the whole spectral range. $p 3 d$, an open-source data reduction and analysis software (http://p3d.sourceforge.net/, last access 18 May 2016) that was initially developed for astronomical purposes (Sandin at al., 2010), has been customized for that project to 


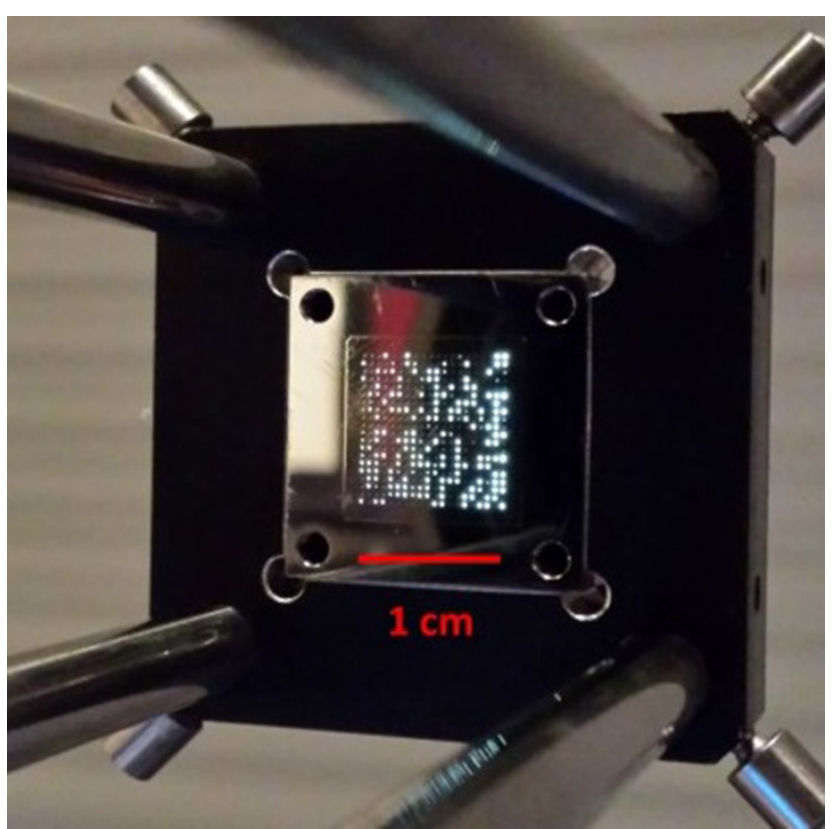

Figure 2. (Reprinted from Schmälzlin et al., 2015): image acquisition head with a $20 \times 20$ fiber matrix. For demonstration purposes of this picture the fibers are partially illuminated at the distal end of the fiber bundle.

calculate the spectra from the raw CCD image and to correlate them with the source position at the two-dimensional fiber array. Finally, a data cube is generated, which contains all spectral and spatial information for further data processing. The fiber bundle contains 400 step index multimode fibers $(110 \mu \mathrm{m}$ core $)$, forming a $20 \times 20$ matrix at the image acquisition side (Fig. 2). Hence, a spectrally resolved square image composed of 400 pixels can be recorded within one single exposure. The pitch between the fiber core centers is $0.5 \mathrm{~mm}$, which is an acceptable tradeoff between resolution and crosstalk with regard to the scattering properties of skin samples.

\subsection{Optics for the acquisition of large-area Raman images}

The use of fiber bundle coupled IFS for imaging Raman spectroscopy was already reported (Ma and Ben-Amotz, 1997; Okuno and Hamaguchi, 2010; Roth et al., 2012; Stewart et al., 2012; Schmälzlin et al., 2014, 2015; Brückner et al., 2015). However, previous setups were designed to examine samples with microscopic dimension, e.g., cells or tiny crystal fragments. Here, we present a setup that is capable of acquiring Raman images from samples with a size of up to $1 \mathrm{~cm}^{2}$, which is the typical size of potentially cancerous skin patches (Reble et al., 2014). Two optic setups were developed. Acquisition optics 1 (AO1) allows recording of Raman images from square surfaces with $3.6 \mathrm{~mm}$ edge length. Acquisition optics $2(\mathrm{AO} 2)$ is even suitable for a square area

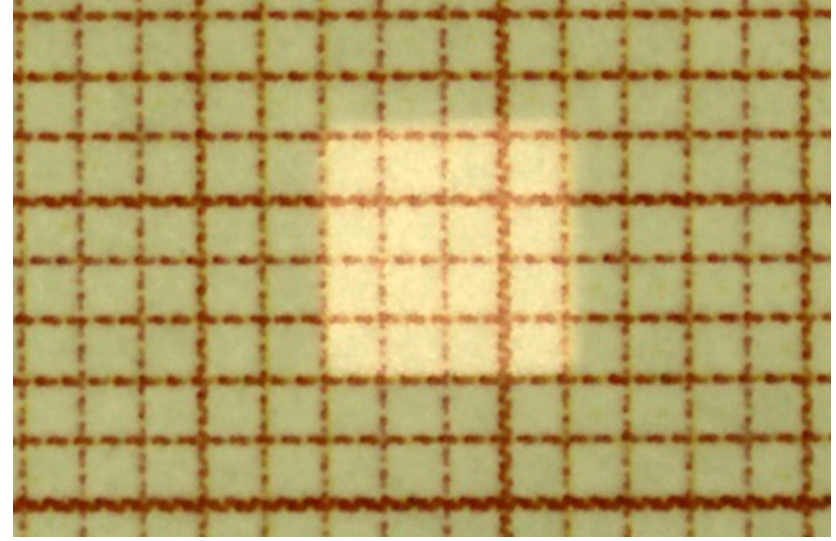

Figure 3. Excitation spot generated with $\mathrm{AO} 1$ at the sample plane. The pitch of the scaled paper is $1 \mathrm{~mm}$.

with $1 \mathrm{~cm}$ edge length. In order to receive large-area Raman images within one single exposure and without scanning, it is favorable to illuminate the whole area of interest as uniformly as possible with the excitation laser. This was performed by feeding the laser light with a square core fiber (F\&T Fibers and Technology GmbH, Germany, VISIR, square core with $600 \mu \mathrm{m}$ edge length). Highly multimode square core fibers, which have been designed initially for laser material processing, shape the incoming Gaussianlike beam to a top-hat intensity distribution (Brückner et al., 2015). Figure 3 shows the resulting square and uniform excitation light spot generated by AO1 at a millimeter-scaled paper. The spot is somewhat larger than the actual image field of the sample to exclude the effect of possible intensity inequalities at the spot's borders.

Figure 4 shows a schematic drawing and a photo of AO1. The excitation laser light $(785 \mathrm{~nm})$ is fed via the square core glass fiber, passes a collimator and a $785 \mathrm{~nm}$ clean-up filter (Edmund Optics) and is finally guided to the sample by use of a $45^{\circ}$ dichroic mirror (Semrock, Di02-R785-25x36) that is transparent for Stokes-shifted Raman signals. An achromatic doublet $(f=50 \mathrm{~mm})$ focuses the laser light on the sample and collects the Raman scattering. A further dichroic mirror (755 nm long pass, Semrock, USA, FF757-Di01-25x36) takes visible light from the sample and guides it to a camera that is used to position and focus the sample. A $785 \mathrm{~nm}$ long pass filter (Semrock, USA, BLP01-785R-25) blocks the excitation laser light and the Rayleigh scattering. A further achromatic doublet ( $f=125 \mathrm{~mm}, \mathrm{NA} \approx 0.1$ ) focuses the image of the sample, strictly speaking the arising Raman signals, onto the surface of the fiber matrix. For convenient positioning of the sample, an XY stage linked to a micrometer lifting table was used. The measurement head was mounted below the opening of the stage plate.

A weak point in the design of $\mathrm{AO} 1$ is the poor coupling efficiency at the side of the fiber matrix. The fiber matrix has an area of $100 \mathrm{~mm}^{2}$; however, only $4 \mathrm{~mm}^{2}$ consist of fiber 

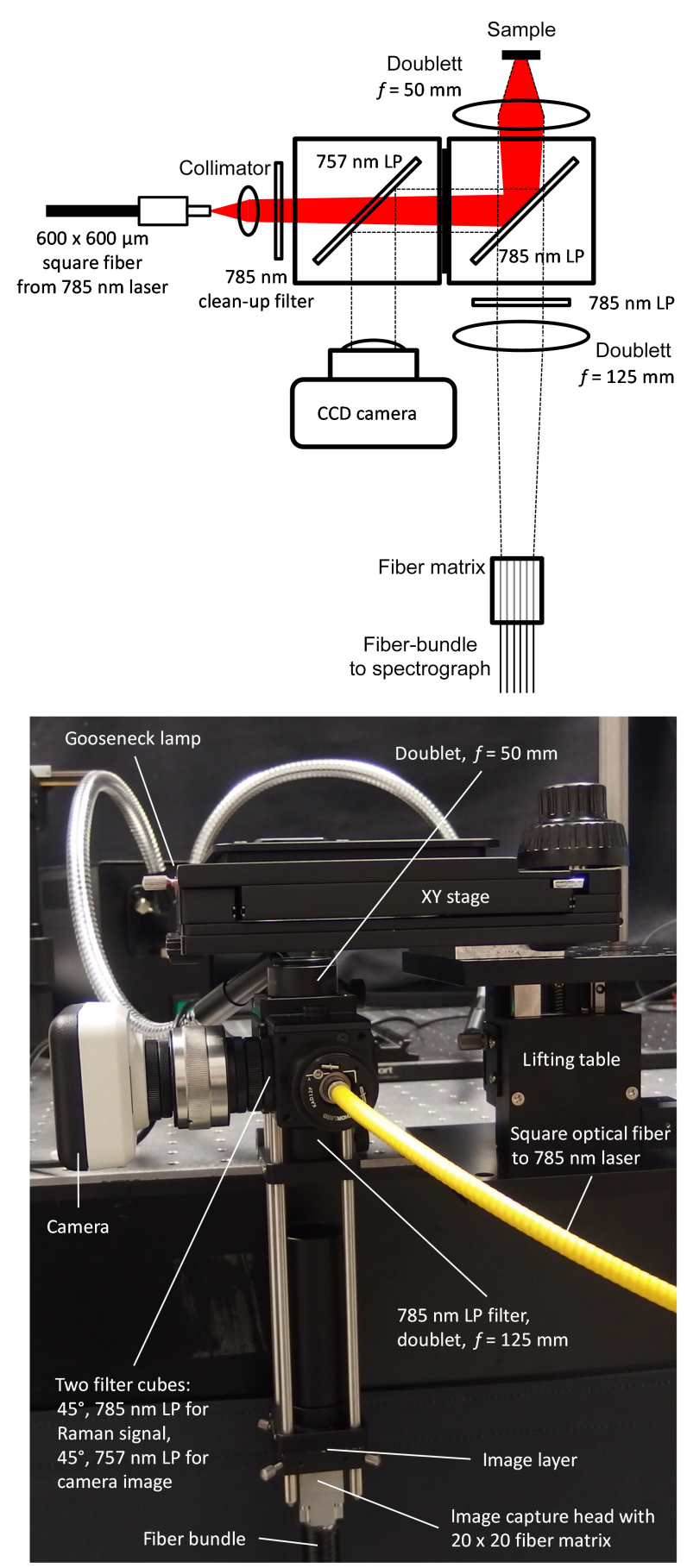

Figure 4. Design and photo of image acquisition optic AO1 suitable for a square image field with $3.6 \mathrm{~mm}$ side length.

cores. To improve the situation, a further optic unit (AO2) with a first microlens array (MLA; Advanced Microoptic Systems, Germany, APO-Q-P500(D500)-AR0.58) in front of the fiber matrix was designed. The MLA consists of $24 \times 24$ photolithographical-manufactured, fused silica microlenses with $1.3 \mathrm{~mm}$ focus lengths, $0.5 \mathrm{~mm}$ pitch, and $0.5 \mathrm{~mm}$ lens

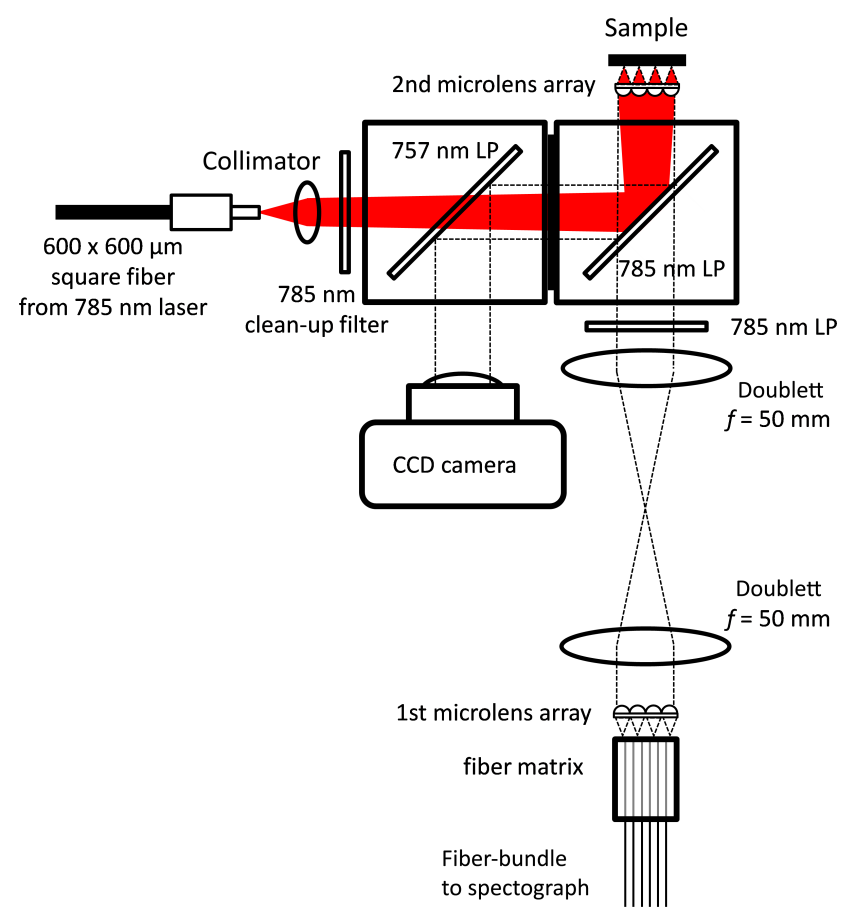

Figure 5. Design of the microlens-based image acquisition optic $\mathrm{AO} 2$ suitable for a square image field with $1 \mathrm{~cm}$ side length.

diameters. The clear aperture of each lens is $0.45 \mathrm{~mm}$, resulting in a fill factor of $65 \%$. The flat side of the MLA points towards the fiber matrix; $20 \times 20$ lenses are actively used to focus the signal into the fiber cores and to reduce losses at blind areas of the fiber matrix. Figure 5 shows a schematic drawing of AO2. A second MLA of the same type was used to increase the image field at the sample side to $10 \mathrm{~mm} \times 10 \mathrm{~mm}$, which was an important objective for a future skin cancer detection application.

The flat side of the second MLA points towards the sample. Figure 6 shows the excitation laser spots at a millimeter scaled paper. A pair of relay lenses guides the Raman signal from the second to the first MLA. Although the realization of $\mathrm{AO} 2$ is still preliminary, first test measurements with $\mathrm{AO} 2$ could be performed successfully.

For biological samples, a vessel with a calcium fluoride bottom is available (Fig. 8). Raman grade calcium fluoride is a suitable slide material since it generates a low background signal.

\subsection{Laser source}

As a light source, a fiber-coupled dual laser light source (Newport, USA, LS-2-7878-FC) that contains two switchable diode lasers with 784.5 and $785.5 \mathrm{~nm}$ was used. The maximum output power of each laser is $500 \mathrm{~mW}$; however, the laser power was normally aligned to $230 \mathrm{~mW}$ measured in the sample plane of AO1. This corresponds to a power density of $14 \mathrm{~mW} \mathrm{~mm}^{-2}$ in the case of a uniformly illumi- 


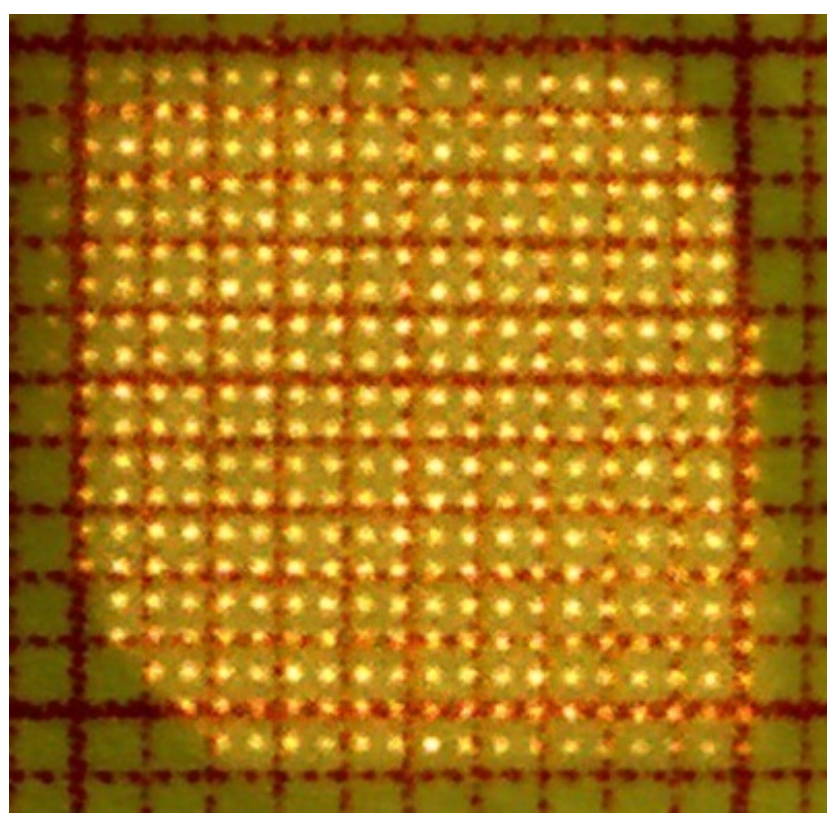

Figure 6. Spots of the excitation laser at about $0.8 \mathrm{~mm}$ distance from the flat surface of the MLA. The pitch of the scaled paper is $1 \mathrm{~mm}$. The slanted shadings at the lower left and at the upper right are due to distance holders.

nated square with $4.1 \mathrm{~mm}$ side length. An estimation of the power density at the sample plane of $\mathrm{AO} 2$ is done as follows: at a $100 \mathrm{~mm}^{2}$ sample field, i.e., in the field of 400 spots, a total laser power of $243 \mathrm{~mW}$ is measured. According to calculations (Zemax LLC, USA, Opticstudio), the predicted diameter of one single laser spot under perfect alignment conditions is $0.01 \mathrm{~mm}$. The actual value is roughly $0.05 \mathrm{~mm}$. Thus, 400 spots illuminate a total area of $0.8 \mathrm{~mm}^{2}$, resulting in about $300 \mathrm{~mW} \mathrm{~mm}^{-2}$ power density within a spot. Taking into account the $65 \%$ fill factor of the MLA, i.e., excluding the light amount passing between the individual lenses, the power density is reduced to $195 \mathrm{~mW} \mathrm{~mm}^{-2}$. It is worth noting that common single channel Raman microscopes work with far higher power densities. For instance, the microscope used in Reble et al. (2014) applies $70 \mathrm{~mW}$ to a circular spot with $0.16 \mathrm{~mm}$ diameter, which corresponds to $3.5 \mathrm{~W} \mathrm{~mm}^{-2}$.

\subsection{SERDS measurements}

The elimination of background fluorescence is a challenge in the field of Raman spectroscopy. In principle, an increase of the excitation wavelength helps to reduce background fluorescence. However, Raman scattering intensity also decreases, since it depends on the fourth power of the excitation frequency. The $785 \mathrm{~nm}$ excitation wavelength is often used for organic samples, since it is an acceptable tradeoff between fluorescence and Raman intensity. Furthermore, a wide range of detectors, lasers, and optical filters is available for this wavelength. Nevertheless, the amount of fluo- rescence may exceed the Raman signal intensity by several orders of magnitude. Various methods are described to remove the fluorescence background (Wei et al., 2015). Most common are post-processing computational methods, e.g., polynomial fitting and subsequent subtracting of the background. A more advanced background removal method is shifted excitation Raman difference spectroscopy (SERDS; Sowoidnich and Kronfeldt, 2012). It is based on the fact that Raman signals always appear relative to the excitation laser wavelength, but fluorescence emission occurs at fixed wavelengths. For SERDS, the Raman spectra of a sample are recorded twice with two slightly different excitation wavelengths (here 784.5 and $785.5 \mathrm{~nm}$, respectively) followed by the application of an appropriate subtraction and transformation procedure. Finally, background-free Raman spectra are reconstructed. The used algorithm is shown in Schmidt et al. (2014).

\subsection{Samples}

The capability of the described setups was demonstrated with the following samples: the Aspirin Effect pain reliever, a piece of silicon, a EUR 10 bank bill, the cut face of a porcine ear, porcine skin, and a piece of brown sugar dissolving in water.

\section{Result and discussions}

\subsection{Aspirin Effect}

Aspirin Effect (Bayer Bitterfeld GmbH, Germany) is a pain reliever tablet consisting of rod-like granules with about $1 \mathrm{~mm}$ length and relatively unshaped fragments. According to the package information sheet, it contains the active agent acetylsalicylic acid, D mannitol, sodium hydrogen carbonate, sodium dihydrogen citrate, ascorbic acid, citric acid, flavors, and the aspartame sweetener. A small spatula tip of a granule was put on a $1 \mathrm{~mm}$ thick calcium fluoride slide and placed on the XY stage over AO1. The Raman spectra were recorded at 784.5 and $785.5 \mathrm{~nm}$ excitation wavelength. The measurement times were $1 \mathrm{~s}$ for each wavelength. The readout time of the full CCD chip was chosen to be $10 \mathrm{~s}$, which is the shortest possible without binning. Thus, the effective measurement time for a complete data set was $22 \mathrm{~s}$. For all further experiments a $10 \mathrm{~s}$ read-out time was chosen as well. Figure 7 shows the camera image, the SERDS spectra at two picked positions and the Raman image. The Raman image illustrates the intensity distribution of the Raman signal at $1606 \mathrm{~cm}^{-1}$ that is characteristic of acetylsalicylic acid. Obviously, the acetylsalicylic acid agent is present in the form of the fine powder. The rod-like granules contain no active agent. 

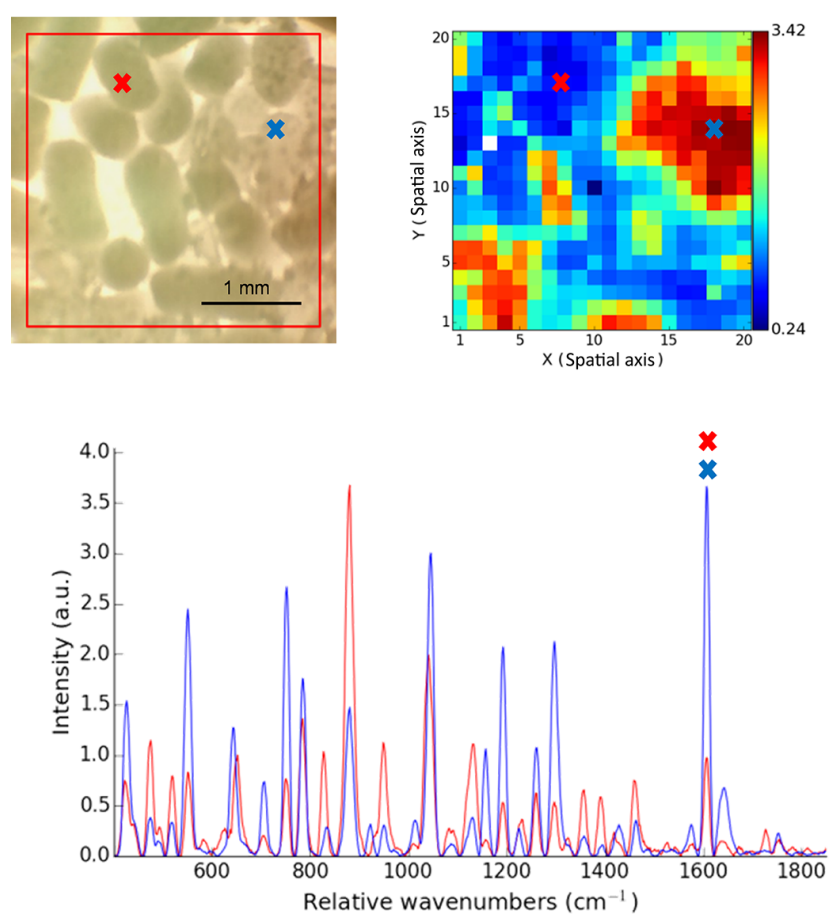

Figure 7. Granules and powder of the Aspirin Effect pain reliever. Upper left: camera picture. Upper right: pseudo color Raman image of the intensity at $1606 \mathrm{~cm}^{-1}$, which corresponds to the distribution of the acetylsalicylic acid agent. Below: corresponding SERDS spectra at positions $*$ and $*$ ( $1 \mathrm{~s}$ recording time per laser).

\subsection{Silicon}

A small silicon plate with $0.66 \mathrm{~mm}$ thickness is used for testing $\mathrm{AO} 2$ and a comparison with $\mathrm{AO} 1$. Pure silicon shows one single Raman signal around $520 \mathrm{~cm}^{-1}$ (Parker, 1967). To determine the optimal distance from the surface of the microlens array, the silicon plate was put in a tilted position on the top of AO2. Figure 8 left shows the silicon plate in front of the microlens array. At the upper right, the silicon plate touches the head of a turned screw. At this point of support the distance from the MLA surface is $d_{1}=1.9 \mathrm{~mm}$. At the lower left, the silicon plate touches the corner MLA surface $\left(d_{0}=0 \mathrm{~mm}\right)$. Figure 8 right shows the intensity distribution of the silicon Raman peak at $522 \mathrm{~cm}^{-1}$. The Raman spectra are recorded at $784.5 \mathrm{~nm}$ for $20 \mathrm{~s}$. The rolling circle method (Brandt et al., 2006) was applied to remove fluorescence background arising from the optics. Obviously, pixels located at the borders are effected by vignetting. The microlens rows at the right and the lower borders are close to the contact area of the holder plate. The given cutting lines of the MLA and the limited adjustment possibilities of $\mathrm{AO} 2$ did not allow for a more favorable position. Furthermore, the visual impression of the image of the laser spots (Fig. 8) suggests that the excitation intensities are somewhat lower at the border lines. Apart from this limitation the shape of the silicon piece is reproduced very well. Around the spatial position
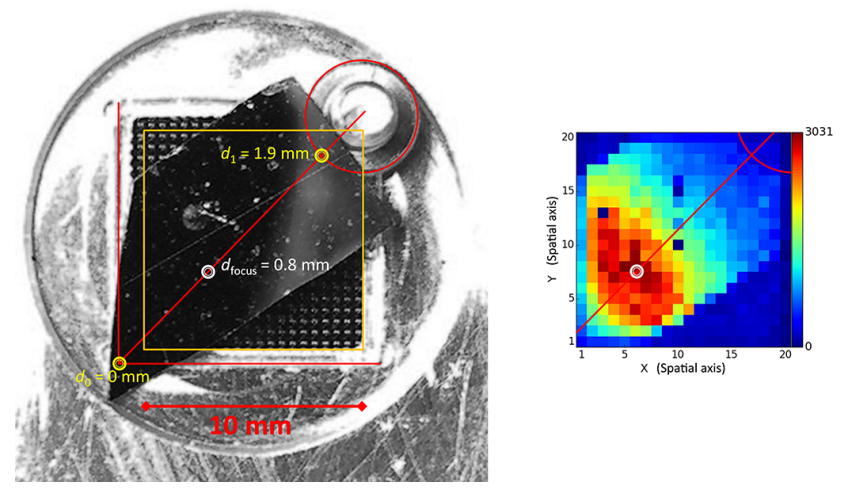

Figure 8. A tilted silicon piece is put in front of the sample-side MLA of AO2 (left). The distant-dependent distribution of the silicon's Raman signal is plotted in pseudo colors (right). Triangulation yields an optimal sample distance $d_{\text {focus }}=0.8 \mathrm{~mm}$, showing the highest Raman intensity.

$(X / Y)=(6 / 8)$ there is an obvious distance-dependent signal maximum of about 3000 counts per pixel. The maximum position was transferred to camera picture and the distances to the two support points were measured. Triangulation yields a distance of $d_{\text {focus }}=0.8 \mathrm{~mm}$ that points to the highest signal intensity. The experiment was repeated by using Raman grade calcium fluoride windows with thicknesses of $0.6,0.7$, 0.8 and $1.0 \mathrm{~mm}$, respectively. The windows were placed as distance holders between the MLA and the silicon plate. The maximum average Raman signal intensity of the silicon piece was detected when using a $0.8 \mathrm{~mm}$ window. Computer simulations of the AO2 setup (Zemax LLC, USA, Opticstudio) predict an optimal distance of $0.6 \mathrm{~mm}$ in the case of an air gap between MLA surface and sample and $1.0 \mathrm{~mm}$ using a calcium fluoride window as spacer. The differences from the experiment values are likely due to adjustment deficits of $\mathrm{AO} 2$. Simulations show that the focus at the sample side depends much on the fine adjustment of the distal MLA. However, a precise alignment in the micrometer range and an accurate control of the actual positions of the optical components cannot be realized with the current $\mathrm{AO} 2$ setup.

For comparison, the silicon plate was also examined with AO1. The silicon plate was positioned at the focal plane of AO1 and the Raman spectra were recorded with an excitation wavelength of $784.5 \mathrm{~nm}$ and an exposure time of $20 \mathrm{~s}$. The settings of the diode laser unit were the same as for the measurement with $\mathrm{AO} 2$. The average intensity of the Raman signal achieved with AO1 was 1000 counts per pixel. Thus, the MLA-based AO2 provides 3 times more counts per pixel than AO1. To estimate the amount of improvement, the following considerations were made: the image field sizes captured with one single fiber of the array are $13 \mathrm{~mm}^{2} / 400 \times 0.04=1.3 \times 10^{-3} \mathrm{~mm}^{2}$ in the case of AO1 (taking into account that without an MLA only $4 \%$ of the projected signal couples into the fibers of the matrix; see 

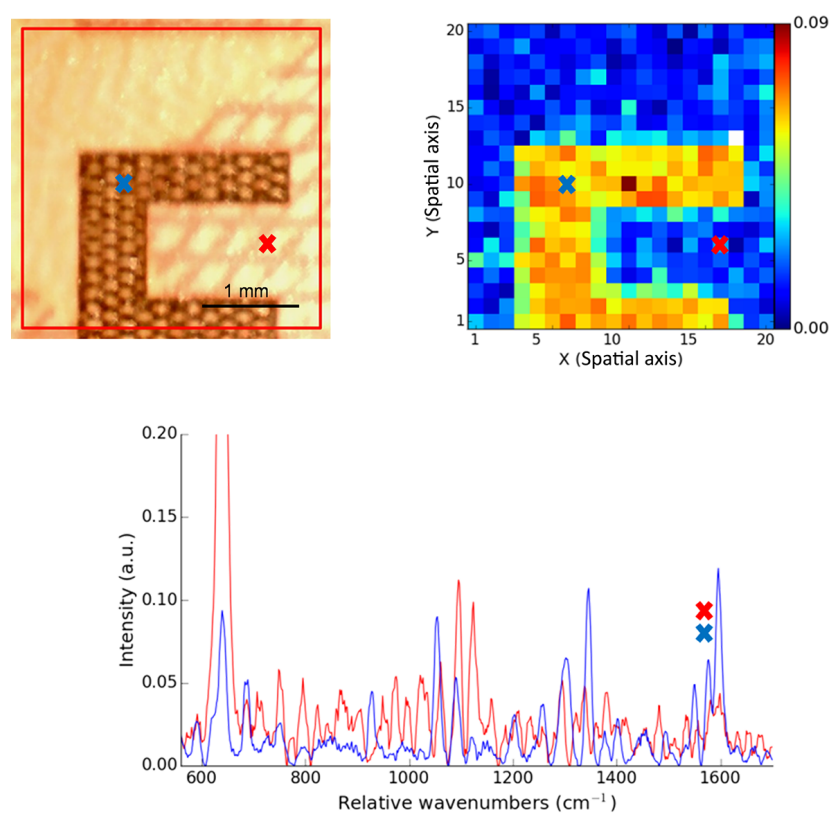

Figure 9. Part of the letter "E" printed on a EUR 10 bill. Upper left: camera picture. Upper right: pseudo color Raman image of the intensity at $1576 \mathrm{~cm}^{-1}$, which corresponds to the distribution of the printing ink of the "E". Below: corresponding SERDS spectra at positions $*$ and $*$ (10 s recording time per laser). The measurements were performed with AO1.

Sect. 2.3) and $100 \mathrm{~mm}^{2} / 400=0.25 \mathrm{~mm}^{2}$ in the case of $\mathrm{AO} 2$ (assuming ideal MLAs). Within the $13 \mathrm{~mm}^{2}$ image field of AO1, an excitation laser power of $295 \mathrm{~mW}$ was measured; i.e., the excitation power per image field of one single fiber of $\mathrm{AO} 1$ is $295 \mathrm{~mW} / 13 \mathrm{~mm}^{2} \times 1.3 \times 10^{-3} \mathrm{~mm}^{2}=0.030 \mathrm{~mW}$. For $\mathrm{AO} 2$, an excitation laser power of $243 \mathrm{~mW}$ at an area of $100 \mathrm{~mm}^{2}$ was measured, leading to an excitation power per fiber of $243 \mathrm{~mW} / 400=0.61 \mathrm{~mW}$. Consequently, an ideal $\mathrm{AO} 2$ should provide twentyfold more Raman signal per pixel than AO1, meaning that if 1000 counts per pixel are achieved with $\mathrm{AO} 1$, an ideal $\mathrm{AO} 2$ should provide 20000 counts per pixel with the same sample in focus. However, the real AO2 shows imperfections that can be quantified. Intermediate space between the single lenses leads to an MLA fill factor of $65 \%$, reducing the effective power of the excitation light and therefore decreasing the number of expectable counts per pixel to $20000 \times 0.65=13000$. It is worth noting that the Raman signal of the silicon only arises from the focus points of the microlenses, i.e., in the places of the spots of the excitation laser. Thus, the fill factor should not affect the collection of the Raman signal, at least not in the case of non-scattering samples like silicon.

Furthermore, the two MLAs do not have antireflection coatings. Back reflections at four surfaces reduce the expectable counts per pixel to $13000 \times(0.96)^{4}=11000$. The real $\mathrm{AO} 2$ provides 3000 counts per pixel. This implies that in theory $\mathrm{AO} 2$ could be approximately even 4 times better. The
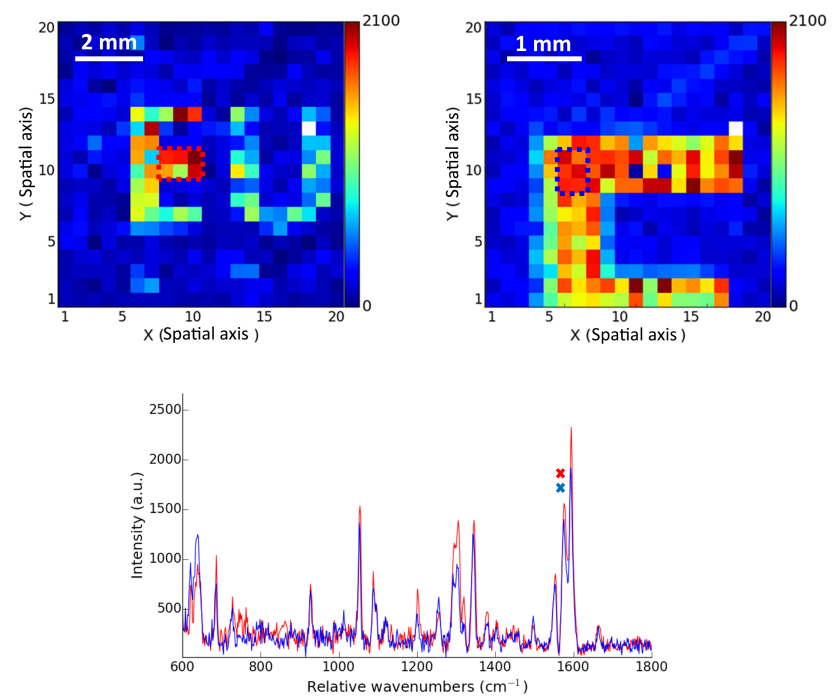

Figure 10. Comparison of AO1 and the MLA-based AO2 by recording Raman images of the lettering "EURO" printed on a EUR 10 bill. Upper left: pseudo color Raman image of a part of the "E" achieved with AO1. Upper right: pseudo color Raman image of the letters "EU" achieved with AO2. Both Raman images are based on the intensity at $1576 \mathrm{~cm}^{-1}$, which corresponds to the distribution of the printing ink. Below: average Raman spectra received from six pixels with AO1 (blue curve) and AO2 (red curve), respectively. The positions of the binned pixels are outlined with squares in the pseudo color images.

difference is most likely due to the limited imaging qualities of the MLAs and the suboptimal alignment of AO2. It should be emphasized that the current version of $\mathrm{AO} 2$ is still a laboratory sample, the optical components of which are difficult to align in a micrometer range.

\subsection{EUR 10 bank bill}

A EUR 10 bank bill was placed on a $1 \mathrm{~mm}$ thick calcium fluoride slide and fixed on the XY stage over AO1. A part of the letter "E" of the inscription "EURO" on the front side is focused. Figure 9 shows the camera image, the Raman image, and the SERDS spectra at two positions. One position $*$ was picked within, the other $*$ outside of the "E". The intensity of the line at $1576 \mathrm{~cm}^{-1}$ seems to be characteristic of the printing ink of the "E" and was used to generate the pseudo color Raman image.

For comparison, the measurement was also performed with MLA-based AO2. The part of the bill with the writing "EURO" was placed over the sample-side MLA. A Raman grade calcium fluoride window was used as a spacer. Its thickness was $0.7 \mathrm{~mm}$; thus, the focus layer of the excitation light was just outside the window body. The measurement was performed with only one laser $\left(784.5 \mathrm{~cm}^{-1}\right)$. Hence, no SERDS spectra were evaluated. The fluorescence background was removed by means of the rolling circle method 


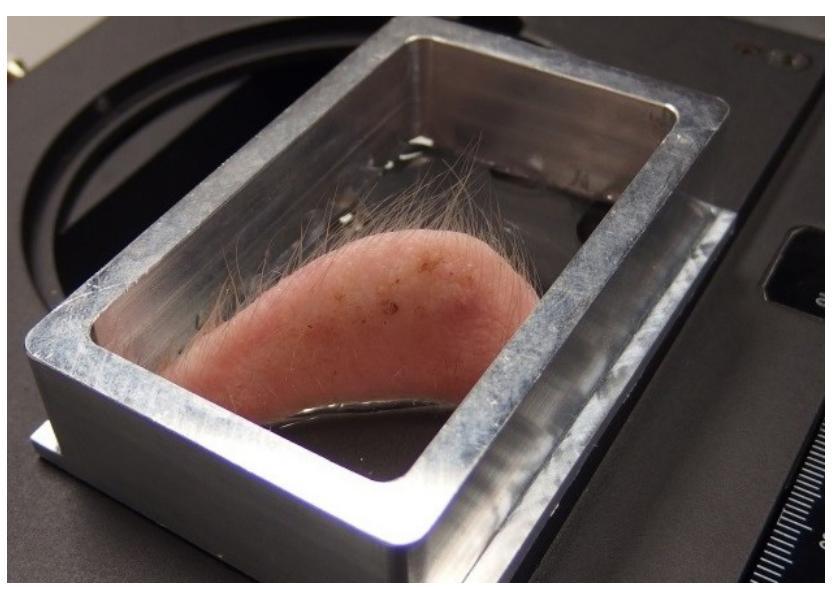

Figure 11. Porcine ear with the cut face down at the calcium fluoride plate of the Raman sample chamber.

(Brandt et al., 2006). The Raman spectra of the letter "E" received with $\mathrm{AO} 1$ at $784.5 \mathrm{~cm}^{-1}$ previously were likewise background-corrected with the rolling circle method. The measurement time was $10 \mathrm{~s}$ in both cases. The power output of the laser was not altered when changing from $\mathrm{AO} 1$ to $\mathrm{AO} 2$. Figure 10 shows the results. The pseudo color Raman images show the letters "EU" recorded with AO2 and the part of the "E" recorded with AO1 related to the intensity of the highest Raman peak of the printing ink at $1576 \mathrm{~cm}^{-1}$. Applying the SERDS algorithm leads to arbitrary units, whereas the rolling circle method retains "counts" as a unit. Thus, the intensity scales in Figs. 9 and 10 are different. The white square at $(18 / 3)$ is due to a broken fiber of the bundle. The Fig. 10 bottom shows the corresponding Raman spectra. The blue curve corresponds to AO1, the red one to AO2. The curves are the averages received from the Raman spectra of, respectively, six individual pixels. The average was taken to reduce the noise. The used pixels marked by rectangles are plotted into the pseudo color images.

$\mathrm{AO} 2$ provides about $10 \%$ higher intensity per pixel than AO1. Indeed, the MLAs of AO2 improve the signal strength. However, the gain is much lower than for the silicon plate (see Sect. 3.2). This is likely due to the comparatively high scattering of the paper. The scattering of the paper increases the diameters of the excitation laser spots (Fig. 6). Due to the $65 \%$ fill factor of the MLA, Raman signals arising from the borders of the laser spots that are increased by scattering samples are not collimated. Furthermore, using a calcium fluoride window affects the light path and thus the alignment conditions of all optical components. Possibly, the thin bill was not exactly in the focus plane.

\subsection{Cut surface of a porcine ear}

From a fresh porcine ear, i.e., the ear was not scalded after slaughtering, the upper part was cut with a scalpel and placed
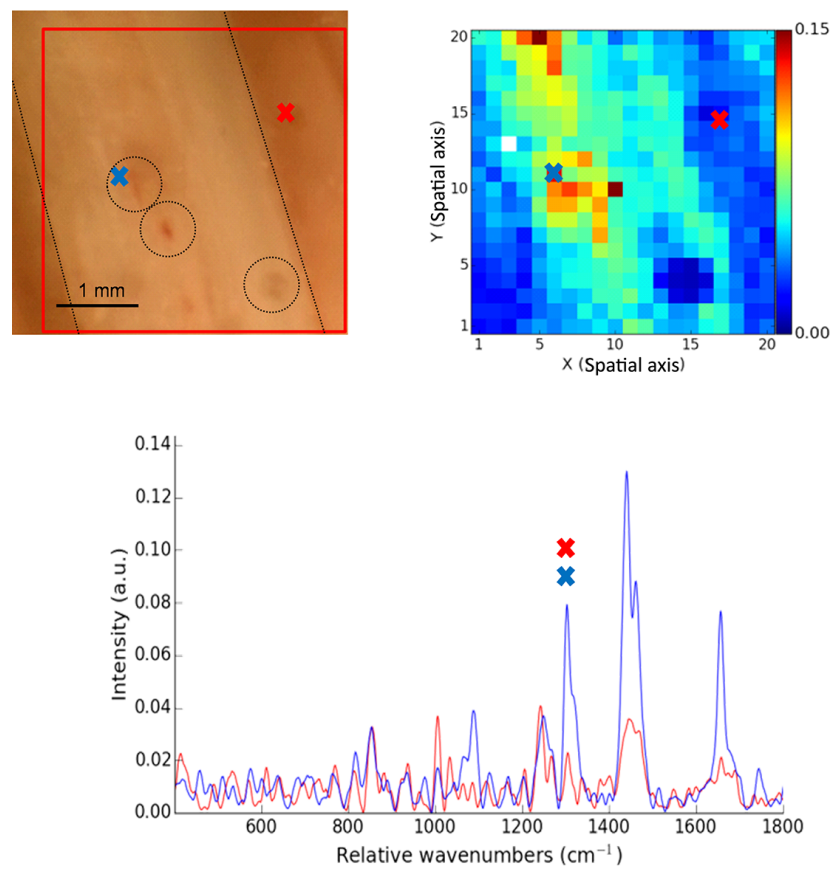

Figure 12. Cut face of a porcine ear. Upper left: camera picture. Upper right: pseudo color Raman image of the intensity at $1303 \mathrm{~cm}^{-1}$ (lipid band). Below: corresponding SERDS spectra at positions $*$ and $*$ (60 s recording time per laser).
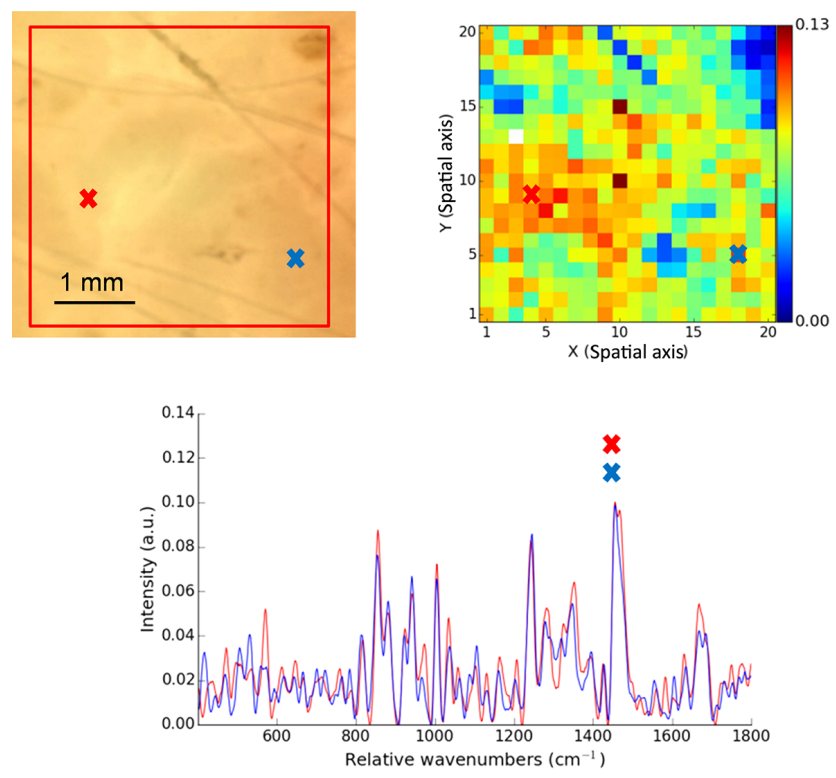

Figure 13. Porcine skin. Upper left: camera picture. Upper right: pseudo color Raman image of the intensity at $1455 \mathrm{~cm}^{-1}$. Below: corresponding SERDS spectra at positions $\star$ and $\star$ (60 s recording time per laser).

face down at the calcium fluoride bottom of a specimen vessel (Fig. 11). The cut face was centered and focused. The Raman spectra were recorded at 784.5 and $785.5 \mathrm{~nm}$ exci- 

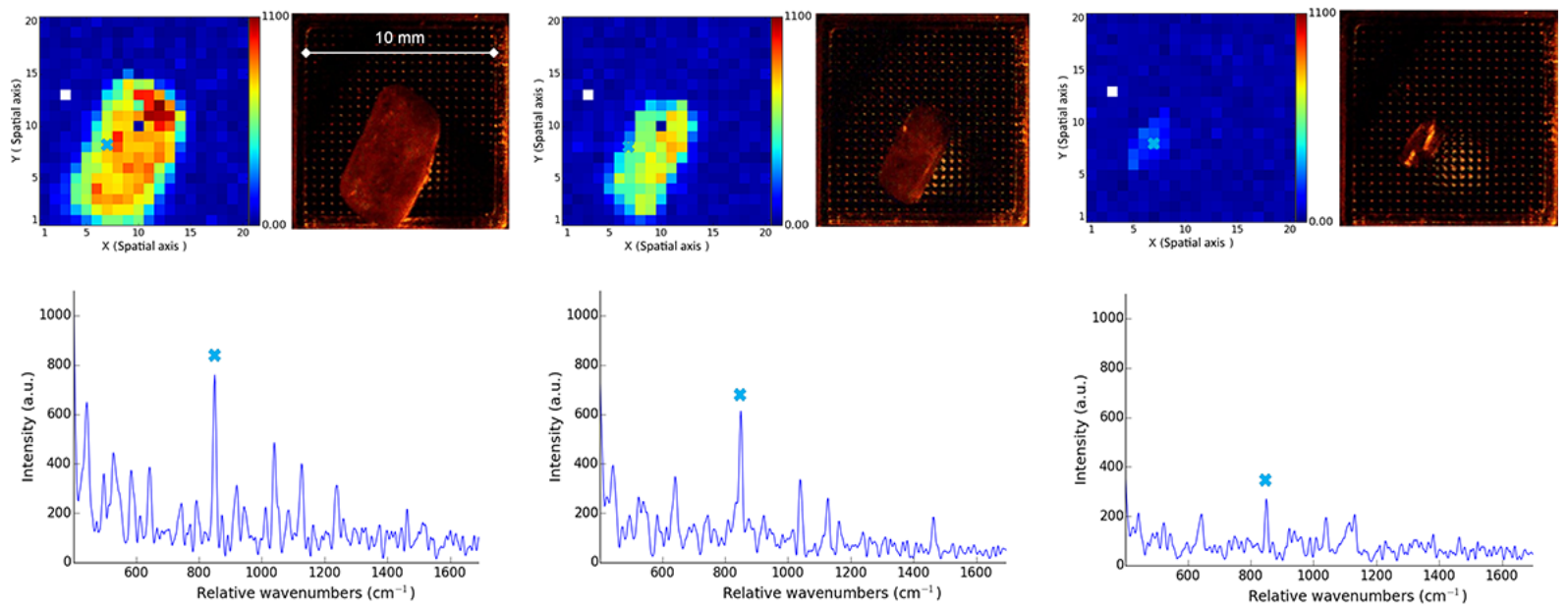

Figure 14. Piece of sugar in water. The dissolving process is tracked using quasi real-time Raman imaging: first frame (left, $0 \mathrm{~s}$ ), 20th frame (center, $390 \mathrm{~s}$ ), and 40th frame (right, $780 \mathrm{~s}$ ). The pseudo colors illustrate the intensity at $850 \mathrm{~cm}^{-1}$. Below the corresponding Raman spectra at the spatial position $(X / Y)=(7 / 8)$ are shown.

tation wavelengths for $60 \mathrm{~s}$ each. In the middle of the cross section there is obviously chondral tissue. The camera image (Fig. 12) shows it as a $3 \mathrm{~mm}$ broad and light vertical stripe that is slightly tilted (region between the dotted lines). Around the $(X / Y)$ positions $(7 / 10)$ and $(9 / 7)$ there are reddish spots and at $(16 / 3)$ there is a dark spot (marked by the dotted circle in the camera image). The position of the chondral tissue as well as those of the reddish and dark spots were retrieved within the Raman image that corresponds to the intensities at $1303 \mathrm{~cm}^{-1}$ (lipid band).

\subsection{Porcine skin}

A piece of porcine skin is placed with the surface down on the $1 \mathrm{~mm}$ calcium fluoride slide and focused. Porcine skin is morphologically similar to human skin (Darvin et al., 2014; Tfaili et al., 2012), and shall be used as a model system to optimize the spectrograph with regard to future human skin cancer diagnostics. To avoid drying of the skin, some water was supplied on the slide and the specimen vessel closed with a lid. The Raman spectra were recorded at 784.5 and $785.5 \mathrm{~nm}$ excitation wavelengths for $60 \mathrm{~s}$ each. The results are shown in Fig. 13. The Raman image was generated by using the Raman intensity at $1455 \mathrm{~cm}^{-1}\left(\mathrm{CH}_{2}\right.$ bending vibrations of proteins). The structures that are visible in the camera image also find themselves in the Raman image in detail. The pigmented spot in the upper right corner as well as the obviously somewhat dirty hair that passes diagonally the upper right quadrant of the image field are very visible. The weak pigmented spots at (3/15) and (13/5) are also identifiable in the Raman image. The obtained Raman spectra are similar, with Raman spectra of porcine skin obtained using another Raman microscope (Zhu et al., 2015).

\subsection{Piece of brown sugar}

With regard to future real-time Raman imaging of samples that change over time, a software script was applied that allows automatic recording of Raman spectra sequences. As a model system, the dissolution of a piece of brown sugar in water was chosen. The sugar was put into a water-filled dish at the top of $\mathrm{AO} 2$ and the Raman measurements were started. For each frame, the recording time was $10 \mathrm{~s}$, followed by $10 \mathrm{~s}$ read-out time. Thus, the effective temporal resolution was $20 \mathrm{~s}$. To track the dissolving process of the sugar in quasi real time, a total number of 60 frames was recorded. Figure 14 shows the situation at 0,390 and $780 \mathrm{~s}$. At the top Raman and camera images, at the bottom the corresponding Raman spectra at the spatial position $(X / Y)=(7 / 8)$ are displayed. The rolling circle method (Brandt et al., 2006) was applied to remove background. The spectra reveal that the sugar piece consists of sucrose (Brizuela et al., 2012). The pseudo color plots illustrate the intensity of the strongest Raman signal at $850 \mathrm{~cm}^{-1}\left(\mathrm{CH}_{2}\right.$ twisting $)$. The Raman images perfectly match the shape and position of the sugar piece shown at the camera images.

The bottleneck with regard to the temporal resolution is the long read-out time of the CCD chip. Unfortunately the available camera system always requires an entire read-out of the CCD chip. However, a new camera system that allows one to define custom read-out regions and thus shorter down times is currently under construction.

\section{Conclusions}

The setup described in this paper allows the recording of spatially resolved large-scale (up to $100 \mathrm{~mm}^{2}$ ) Raman images without a scanning procedure. The exposure times to receive 
the fill spectral and spatial information were $120 \mathrm{~s}$ (cut face of porcine ear and porcine skin), $20 \mathrm{~s}$ (silicon and EUR 10 bill), $10 \mathrm{~s}$ (sugar) and $2 \mathrm{~s}$ (Aspirin Effect), which corresponds to $300,50,25$, and $5 \mathrm{~ms}$, respectively, per pixel. The readout time of the CCD chip was $10 \mathrm{~s}$. To receive backgroundfree Raman spectra via SERDS, two successive exposures and read-outs are required. The pseudo color Raman images display the intensities of selected bands of the Raman spectra at the corresponding spatial positions. Even with this quite ordinary analysis method, high-contrast Raman images are gained that correspond well to the structures shown by the camera. The results show great promise for being able to identify cancerous tissue in a next step by using differential analysis.

Author contributions. E. Schmälzlin, B. Moralejo, and M. M. Roth designed and realized the optical setup. E. Schmälzlin, B. Moralejo, D. Bodenmüller, M. E. Darvin, and G. Thiede designed and carried out the experiments. E. Schmälzlin, B. Moralejo, and M. E. Darvin analyzed data. E. Schmälzlin and B. Moralejo prepared the manuscript. All authors have revised the study critically and approved submission.

Acknowledgements. The authors acknowledge support from the German Federal Ministry of Education and Research (BMBF) VIP program, grant no. 03V0843, and the Zwanzig20 program, grant no. 03ZZ0423. We thank Johannes Schleusener, Ingo Gersonde, and Jürgen Helfmann from Laser- und Medizin-Technologie $\mathrm{GmbH}$, Berlin, 14195, Germany, for their assistance by the development of the MLA-based optic unit.

Edited by: G. Gauglitz

Reviewed by: two anonymous referees

\section{References}

Allington-Smith, J.: Basic principles of integral field spectroscopy, New Astron. Rev., 50, 244-251, doi:10.1016/j.newar.2006.02.024, 2006.

Bacon, R., Bauer, S.-M., Bower, R., Cabrit, S., Cappellari, M., Carollo, M., Combes, F., Davies, R. L., Delabre, B., Dekker, H., Devriendt, J., Djidel, S., Duchateau, M., Dubois, J.-P., Emsellem, E., Ferruit, P., Franx, M., Gilmore, G. F., Guiderdoni, B., Henault, F., Hubin, N., Jungwiert, B., Kelz, A., Le Louarn, M., Lewis, I. J., Lizon, J.-L., McDermid, R., Morris, S. L., Laux, U., Le Fèvre, O., Lantz, B., Lilly, S., Lynn, J., Pasquini, L., Pecontal, A., Pinet, P., Popovic, D., Quirrenbach, A., Reiss, R., Roth, M. M., Steinmetz, M., Stuik, R., Wisotzki, L., and de Zeeuw, P. T.: The second-generation VLT instrument MUSE: science drivers and instrument design, in: Proc. SPIE 5492, Ground-based Instrumentation for Astronomy, Glasgow, United Kingdom, 21-25 June 2004, 1145, doi:10.1117/12.549009, 2004.

Brandt, N. N., Brovko, O. O., Chikishev, A. Y., and Paraschuk, O. D.: Optimization of the Rolling-Circle Filter for Raman Background Subtraction, Appl. Spectrosc., 60, 288-293, doi:10.1366/000370206776342553, 2006.
Brizuela, A. B., Bichara, L. C., Romano, E., Yurquina, A., Locatelli, S., and Brandán, S. A.: A complete characterization of the vibrational spectra of sucrose, Carbohyd. Res., 361, 212-218, doi:10.1016/j.carres.2012.07.009, 2012.

Brückner, M., Becker, K., Popp, J., and Frosch, T.: Fiber array based hyperspectral Raman imaging for chemical selective analysis of malaria-infected red blood cells, Anal. Chim. Acta, 894, 76-84, doi:10.1016/j.aca.2015.08.025, 2015.

Darvin, M. E., Richter, H., Zhu, Y. J., Meinke, M. C., Knorr, F., Gonchukov, S. A., Koenig, K., and Lademann, $\mathrm{J}$.: Comparison of in vivo and ex vivo laser scanning microscopy and multiphoton tomography application for human and porcine skin imaging, Quantum Electron., 44, 646-651, doi:10.1070/QE2014v044n07ABEH015488, 2014.

Hagen, N., Kester, R. T., Gao, L., and Tkaczyk, T. S.: Snapshot advantage: a review of the light collection improvement for parallel high-dimensional measurement systems, Opt. Eng., 51, 111702, doi:10.1117/1.OE.51.11.111702, 2012.

Li, Q., He, X., Wang, Y., Liu, H., Xu, D., and Guo, F.: Review of spectral imaging technology in biomedical engineering: achievements and challenges, J. Biomed. Opt., 18, 100901, doi:10.1117/1.JBO.18.10.100901, 2013.

Ma, J. and Ben-Amotz, D.: Rapid Micro-Raman Imaging Using Fiber-Bundle Image Compression, Appl. Spectrosc., 51, 18451848, doi:10.1366/0003702971939668, 1997.

Okuno, M. and Hamaguchi, H.: Multifocus confocal Raman microspectroscopy for fast multimode vibrational imaging of living cells, Opt. Lett., 35, 4096-4098, doi:10.1364/OL.35.004096, 2010.

Opilik, L., Schmid, T., and Zenobi, R.: Modern Raman Imaging: Vibrational Spectroscopy on the Micrometer and Nanometer Scales, Annu. Rev. Anal. Chem., 6, 379-398, doi:10.1146/annurev-anchem-062012-092646, 2013.

Parker Jr., J. H., Feldman, D. W., and Ashkin, M.; Raman Scattering by Silicon and Germanium, Phys. Rev., 155, 712-714, doi:10.1103/PhysRev.155.712, 1967.

Reble, C., Gersonde, I., Dressler, C., Helfmann, J., Kühn, W., and Eichler, H. J.: Evaluation of Raman spectroscopic macro raster scans of native cervical cone biopsies using histopathological mapping, J. Biomed. Opt., 19, 027007, doi:10.1117/1.JBO.19.2.027007, 2014.

Roth, M.: Introductory review and technical approaches, in: 3D Spectroscopy in Astronomy, edited by: Mediavilla, E., Arribas, S., Roth, M., Cepa-Norgué, and Sánchez, F., Cambridge University Press, New York, USA, 1-39, 2010.

Roth, M. M., Zenichowski, K., Tarcea, N., Popp, J., Adelhelm, S., Stolz, M., Kelz, A., Sandin, C., Bauer, S.-M., Fechner, T., Popow, E., Roth, B., Singh, P., Srivastava, M., and Wolter, D.: The ERA2 facility: towards application of a fibre-based astronomical spectrograph for imaging spectroscopy in life sciences, in: Proc. SPIE 8450, Modern Technologies in Space- and Ground-based Telescopes and Instrumentation II, Amsterdam, the Netherlands, 1 July 2012, 84501T, doi:10.1117/12.925340, 2012

Sandin, C., Becker, T., Roth, M. M., Gerssen, J., Monreal-Ibero, A., Böhm, P., and Weilbacher, P.: p3d: a general data-reduction tool for fiber-fed integral-field spectrographs, Astron. Astrophys., 515, A35, doi:10.1051/0004-6361/201014022, 2010.

Schmälzlin, E., Moralejo, B., Rutowska, M., Monreal-Ibero, A., Sandin, C., Tarcea, N., Popp J., and Roth M. M.: Raman Imag- 
ing with a Fiber-Coupled Multichannel Spectrograph, Sensors, 14, 21968-21980, doi:10.3390/s141121968, 2014.

Schmälzlin, E., Stolz, M., Moralejo, B., Adelhelm, S., and Roth, M. M.: Imaging multiplex Raman spectroscopy with no need for sequential scanning, Laser. Photonics Rev., 62-65, 2015.

Schmidt, H., Kaiser, P., and Maiwald, M.: European patent EP 2 480868 B1, Patent bulletin 2014/02, 2014.

Sowoidnich, K. and Kronfeldt, H.-D.: Fluorescence Rejection by Shifted Excitation Raman Difference Spectroscopy at Multiple Wavelengths for the Investigation of Biological Samples, ISRN Spectroscopy 2012, 256326, doi:10.5402/2012/256326, 2012.

Stewart S., Priore, R. J., Nelson, M. P., and Treado, P. J.: Raman Imaging, Annu. Rev. Anal. Chem. 5, 337-260, doi:10.1146/annurev-anchem-062011-143152, 2012.

Tfaili, S., Gobinet, C., Josse, G., Angiboust, J.-F., Manfait, M., and Piot, O.: Confocal Raman microspectroscopy for skin characterization: a comparative study between human skin and pig skin, Analyst, 137, 3673-3682, doi:10.1039/c2an16292j, 2012.
Vanderriest, C.: Fiber-Optics Dissector for Spectroscopy of Nebulosities around Quasars and similar Objects, PASP, 92, 858-862, doi:10.1086/130764, 1980.

Wei, D., Chen, S., and Liu, Q.: Review of Fluorescence Suppression Techniques in Raman Spectroscopy, Appl. Spectrosc. Rev., 50, 387-406, doi:10.1080/05704928.2014.999936, 2015.

Zhao, J., Lui, H., Kalia, S., and Zeng, H.: Real-time Raman spectroscopy for automatic in vivo skin cancer detection: an independent validation, Anal. Bioanal. Chem., 407, 8373-8379, doi:10.1007/s00216-015-8914-9, 2015.

Zhu, Y. J., Choe, C.-S., Ahlberg, S., Lademann, J., and Darvin, M. E.: Penetration of silver nanoparticles into porcine skin ex vivo using fluorescence lifetime imaging microscopy, Raman microscopy and surface enhanced Raman microscopy, J. Biomed. Opt., 20, 051006, doi:10.1117/1.JBO.20.5.051006, 2015. 